\title{
A new method to incorporate three-phase power transformer model into distribution system load flow analysis
}

\author{
Rudy Gianto, Purwoharjono \\ Department of Electrical Engineering, Universitas Tanjungpura, Indonesia
}

\begin{tabular}{l} 
Article Info \\
\hline Article history: \\
Received Jan 21, 2021 \\
Revised Apr 4, 2021 \\
Accepted May 27, 2021 \\
\hline
\end{tabular}

Keywords:

Distribution system

Forward/backward sweep

Load flow analysis

Symmetrical component theory

Three-phase transformer

\begin{abstract}
This paper proposes a new and simple method to incorporate three-phase power transformer model into distribution system load flow (DSLF) analysis. The objective of the present work is to find a robust and efficient technique for modeling and integrating power transformer in the DSLF analysis. The proposed transformer model is derived based on nodal admittance matrix and formulated by using the symmetrical component theory. Load flow formulation in terms of branch currents and nodal voltages is also proposed in this paper to enable integrating the model into the DSLF analysis. Singularity that makes the calculations in forward/backward sweep (FBS) algorithm is difficult to be carried out. It can be avoided in the method. The proposed model is verified by using the standard IEEE test system.
\end{abstract}

This is an open access article under the CC BY-SA license.

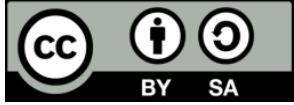

\section{Corresponding Author:}

Rudy Gianto

Department of Electrical Engineering

Universitas Tanjungpura

Prof. Dr. H. Hadari Nawawi St., Pontianak 78124, Indonesia

Email: rudygianto@gmail.com

\section{INTRODUCTION}

Three-phase power transformer is one of the most important components in modern electric power system. The important of this device could probably be ranked with the synchronous machine and the transmission line. Generator transformer, one of the essential types of power transformer, has the function of transforming system voltage from generation level to transmission or sub-transmission level. Another important type of power transformer is distribution substation transformer. This device is usually located at load center and employed to transform the system voltage from transmission level to distribution feeder level. Distribution transformer is then used to provide the final voltage transformation to the customer's load.

Distribution system load flow (DSLF) analysis is carried out to investigate the steady state performances of the distribution system. Electrical quantities such as nodal voltages, substation powers, distribution power flows and losses can be obtained based on the analysis. DSLF techniques can be classified into two broad categories, namely node-based techniques (i.e. Newton-Raphson-based methods) and branchbased techniques (i.e. backward/forward sweeping (FBS)-based methods). In Newton-Raphson-based algorithms [1]-[8], iterative techniques are normally used to obtain the solution. The algorithm will usually start with the initial estimation for the solution. This initial estimation is then updated until the solution with desired accuracy is obtained. Backward/forward sweeping-based methods are spesifically designed to find the solution of DSLF problems [9]-[13]. In searching for the solution, these methods exploit the radial structure of the distribution system. However, forward/backward sweeping method requires special techniques for branch numbering and bus ordering of the distribution system. 
To properly conduct the load flow analysis, steady state modeling of the distribution system components (including the power transformer) is necessary. Several methods have been proposed to model and incorporate three-phase power transformer into load flow analysis [14]-[29]. In [14]-[24], transformer model based on nodal admittance matrices has been implemented to load flow solution methods. However, for some transformer connection types, solution to the load flow problem has been found to be difficult to obtain. This difficulty is mainly caused by the matrix singularity which appears on some transformer connection types.

In [25]-[28], a three-phase power transformer model suitable for load flow analysis is presented. The model has been derived based on sequence components. However, it has been widely acknowledged that classical sequence components representation lacks the efficiency to model the transformer properly since different algorithms have to be used for different transformer connections. Morever, in the model proposed in [25]-[28], some complicated treatments are needed to overcome the singularity problem that appears on some transformer connection types. In [29], Kirchhoff's voltage and current laws are used for each transformer connection for radial distribution system radial load flow analysis. This technique requires a set of separate equations for each transformer connection to be implemented inside the load flow solution process. Therefore, against the above background, the objective of the paper is to find a robust and efficient technique for including three-phase transformer model in DSLF analysis.

In the present work, three-phase power transformer model based on nodal admittance matrix for various transformer connection types are presented. First, transformer model based on nodal admittance matrix in terms of sequence components is developed. Using the symmetrical component theory, the sequence components model is then converted into its equivalent phase components model. To enable incorporating the model into DSLF analysis, the load flow formulation in terms of branch currents and nodal voltages is also proposed in the present paper. In the formulation, sets of nonlinear equations are derived by examining the conditions (i.e. electrical quantities relationships) at every node and branch in the distribution system. The complete set of nonlinear equations is then solved to obtain the solution to the load flow problem.

In the proposed method, the singularity that makes the calculations in FBS algorithm difficult to be carried out can be avoided. As a result, complicated voltage updating procedures as in the FBS-based method, are not required. Moreover, the method proposed in the present paper uses only one algorithm for different transformer connections (i.e. the same algorithm can be applied to all transformer connection types). Therefore, it is more efficient in terms of various transformer connection implementations. Verification of the method using standard IEEE test system is also presented in the present paper.

\section{THREE-PHASE TRANSFORMER MODELING}

\subsection{Transformer model in sequence components}

This section discusses the proposed three-phase transformer model to be integrated into distribution system load flow analysis. The model is first represented in terms of sequence components. Then, in Section 2.2 , it is represented in terms of phase components by using symmetrical component theory. Figure 1 shows a schematic diagram of a three-phase transformer. It can be shown that the mathematical model of the transformer in Figure 1 is given by (1).

$$
\left[\begin{array}{c}
\boldsymbol{I}_{P}^{012} \\
\cdots \\
\boldsymbol{I}_{S}^{012}
\end{array}\right]=\left[\begin{array}{ccc}
\boldsymbol{Y}_{P P}^{012} & \vdots & \boldsymbol{Y}_{P S}^{012} \\
\cdots & \vdots & \cdots \\
\boldsymbol{Y}_{S P}^{012} & \vdots & \boldsymbol{Y}_{S S}^{012}
\end{array}\right]\left[\begin{array}{c}
\boldsymbol{V}_{P}^{012} \\
\cdots \\
\boldsymbol{V}_{S}^{012}
\end{array}\right]
$$

where:

$I_{P}^{012}$ and $\boldsymbol{I}_{S}^{012}$ : transformer primary and secondary sequence currents

$V_{P}^{012}$ and $V_{S}^{012}$ : transformer primary and secondary sequence voltages

$\boldsymbol{Y}_{P P}^{O 12}, \boldsymbol{Y}_{P S}^{O 12}, \boldsymbol{Y}_{S P}^{O 12}$, and $\boldsymbol{Y}_{S S}^{012}:$ transformer sub-matrices

It is to be noted that each of transformer sub-matrices in (1) is of $3 \times 3$ dimension and its value depends on the type of the transformer connection. Table 1 shows the proposed sub-matrix values for common transformer connections. Standard 30-degree connections are assumed for Y-D and D-Y connections. The formulations for matrices $\boldsymbol{Y}_{I}, \boldsymbol{Y}_{I I}, \boldsymbol{Y}_{I I I}$, and $\boldsymbol{Y}_{I V}$ in Table 1 are (2), (3), (4), and (5). 


$$
\begin{aligned}
\boldsymbol{Y}_{I} & =\left[\begin{array}{lll}
y_{t} & 0 & 0 \\
0 & y_{t} & 0 \\
0 & 0 & y_{t}
\end{array}\right] \\
\boldsymbol{Y}_{I I} & =\left[\begin{array}{lll}
0 & 0 & 0 \\
0 & y_{t} & 0 \\
0 & 0 & y_{t}
\end{array}\right] \\
\boldsymbol{Y}_{I I I} & =\left[\begin{array}{ccc}
0 & -y_{t}\left(1 \angle 30^{o}\right) & 0 \\
0 & 0 & -y_{t}\left(1 \angle-30^{o}\right)
\end{array}\right] \\
\boldsymbol{Y}_{I V} & =\left[\begin{array}{ccc}
0 & 0 & 0 \\
0 & y_{t}\left(1 \angle-30^{o}\right) & 0 \\
0 & 0 & y_{t}\left(1 \angle 30^{o}\right)
\end{array}\right]
\end{aligned}
$$

where $y_{t}$ is the transformer leakage admittance.

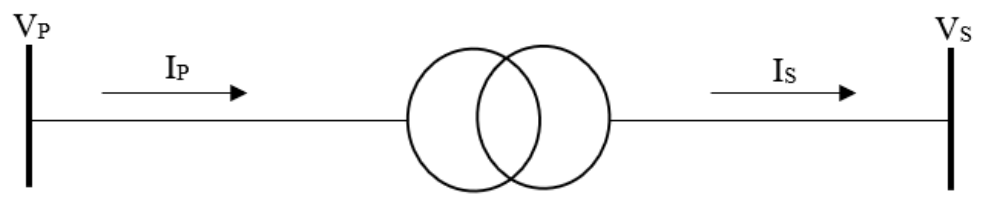

Figure 1. Schematic diagram of three-phase transformer

Table 1. Sub-matrices for common transformer connections

\begin{tabular}{ccccc}
\hline Connections & $\boldsymbol{Y}_{P P}^{O 12}$ & $\boldsymbol{Y}_{P S}^{O 12}$ & $\boldsymbol{Y}_{S P}^{O 12}$ & $\boldsymbol{Y}_{S S}^{012}$ \\
\hline GrY-GrY & $\boldsymbol{Y}_{I}$ & $-\boldsymbol{Y}_{I}$ & $\boldsymbol{Y}_{I}$ & $-\boldsymbol{Y}_{I}$ \\
$\mathrm{D}-\mathrm{D}$ & $\boldsymbol{Y}_{I I}$ & $-\boldsymbol{Y}_{I I}$ & $\boldsymbol{Y}_{I I}$ & $-\boldsymbol{Y}_{I I}$ \\
$\mathrm{Y}-\mathrm{D}$ & $\boldsymbol{Y}_{I I}$ & $\boldsymbol{Y}_{I I I}$ & $\boldsymbol{Y}_{I V}$ & $-\boldsymbol{Y}_{I I}$ \\
$\mathrm{GrY}-\mathrm{D}$ & $\boldsymbol{Y}_{I}$ & $\boldsymbol{Y}_{I I I}$ & $\boldsymbol{Y}_{I V}$ & $-\boldsymbol{Y}_{I I}$ \\
$\mathrm{D}-\mathrm{GrY}$ & $\boldsymbol{Y}_{I I}$ & $\boldsymbol{Y}_{I I I}$ & $\boldsymbol{Y}_{I V}$ & $-\boldsymbol{Y}_{I}$ \\
$\mathrm{Y}-\mathrm{Y}$ & $\boldsymbol{Y}_{I I}$ & $-\boldsymbol{Y}_{I I}$ & $\boldsymbol{Y}_{I I}$ & $-\boldsymbol{Y}_{I I}$ \\
$\mathrm{D}-\mathrm{Y}$ & $\boldsymbol{Y}_{I I}$ & $\boldsymbol{Y}_{I I I}$ & $\boldsymbol{Y}_{I V}$ & $-\boldsymbol{Y}_{I I}$ \\
$\mathrm{GrY}-\mathrm{Y}$ & $\boldsymbol{Y}_{I I}$ & $-\boldsymbol{Y}_{I I}$ & $\boldsymbol{Y}_{I I}$ & $-\boldsymbol{Y}_{I I}$ \\
$\mathrm{Y}-\mathrm{GrY}$ & $\boldsymbol{Y}_{I I}$ & $-\boldsymbol{Y}_{I I}$ & $\boldsymbol{Y}_{I I}$ & $-\boldsymbol{Y}_{I I}$ \\
\hline
\end{tabular}

Note: Gr stands for grounded

\subsection{Transformer model in phase components}

Three-phase load flow formulation proposed in the present work is in phase components. Therefore, the transformer model described in Section 2.1 needs to be converted into its equivalent phase components model. The first step in the conversion process is to rewrite (1) in the forms of two equations as (6) and (7).

$$
\begin{aligned}
& \boldsymbol{I}_{P}^{012}=\boldsymbol{Y}_{P P}^{012} \boldsymbol{V}_{P}^{012}+\boldsymbol{Y}_{P S}^{012} \boldsymbol{V}_{S}^{012} \\
& \boldsymbol{I}_{S}^{012}=\boldsymbol{Y}_{S P}^{012} \boldsymbol{V}_{P}^{012}+\boldsymbol{Y}_{S S}^{012} \boldsymbol{V}_{S}^{012}
\end{aligned}
$$

Then, based on symmetrical component theory, (6) and (7) can be rewritten in phase components as (8) and (9). 


$$
\begin{aligned}
& \boldsymbol{I}_{P}^{a b c}=\boldsymbol{Y}_{P P}^{a b c} \boldsymbol{V}_{P}^{a b c}+\boldsymbol{Y}_{P S}^{a b c} \boldsymbol{V}_{S}^{a b c} \\
& \boldsymbol{I}_{S}^{a b c}=\boldsymbol{Y}_{S P}^{a b c} \boldsymbol{V}_{P}^{a b c}+\boldsymbol{Y}_{S S}^{a b c} \boldsymbol{V}_{S}^{a b c}
\end{aligned}
$$

where:

$\boldsymbol{I}_{P}^{a b c}$ and $\boldsymbol{I}_{S}^{a b c}:$ transformer primary and secondary phase currents

$\boldsymbol{V}_{P}^{a b c}$ and $\boldsymbol{V}_{S}^{a b c}$ : transformer primary and secondary phase voltages

$\boldsymbol{Y}_{P P}^{a b c}, \boldsymbol{Y}_{P S}^{a b c}, \boldsymbol{Y}_{S P}^{a b c}$, and $\boldsymbol{Y}_{S S}^{a b c}$ : transformer sub-matrices in terms of phase components

The transformer sub-matrices $\boldsymbol{Y}_{P P}^{a b c}, \boldsymbol{Y}_{P S}^{a b c}, \boldsymbol{Y}_{S P}^{a b c}$, and $\boldsymbol{Y}_{S S}^{a b c}$ in (8) and (9) are calculated using (10), (11), (12), and (13).

$$
\begin{aligned}
& \boldsymbol{Y}_{P P}^{a b c}=\boldsymbol{A} \boldsymbol{Y}_{P P}^{012} \boldsymbol{A}^{-1} \\
& \boldsymbol{Y}_{P S}^{a b c}=\boldsymbol{A} \boldsymbol{Y}_{P S}^{012} \boldsymbol{A}^{-1} \\
& \boldsymbol{Y}_{S P}^{a b c}=\boldsymbol{A} \boldsymbol{Y}_{S P}^{012} \boldsymbol{A}^{-1} \\
& \boldsymbol{Y}_{S S}^{a b c}=\boldsymbol{A} \boldsymbol{Y}_{S S}^{012} \boldsymbol{A}^{-1}
\end{aligned}
$$

where:

$$
\begin{aligned}
& \boldsymbol{A}=\left[\begin{array}{ccc}
1 & 1 & 1 \\
1 & 1 \angle 240^{\circ} & 1 \angle 120^{\circ} \\
1 & 1 \angle 120^{\circ} & 1 \angle 240^{\circ}
\end{array}\right] \\
& \boldsymbol{A}^{-1}=\left[\begin{array}{ccc}
1 & 1 & 1 \\
1 & 1 \angle 120^{\circ} & 1 \angle 240^{\circ} \\
1 & 1 \angle 240^{\circ} & 1 \angle 120^{\circ}
\end{array}\right]
\end{aligned}
$$

It is to be noted that the sequence components transformer sub-matrices in (14) and (15) are calculated based on the formulas given in Table 1. Equations (8) and (9) are the proposed three-phase transformer mathematical model to be integrated into DSLF analysis. More detail explanation of the model integration will be discussed in the next section.

\section{DSLF ANALYSIS}

\subsection{DSLF formulation}

To enable incorporating three-phase transformer model described in section 2 into the DSLF analysis, a new formulation of DSLF problem is also proposed in the present work. The new DSLF formulation is expressed in terms of branch currents and nodal voltages. Figure 2 shows a section of a distribution network. As distribution lines do not usually have shunt admittances, it has been assumed that the line section has only series admittance. However, if needed, the shunt admittances can be included in the formulation in a straightforward manner. 


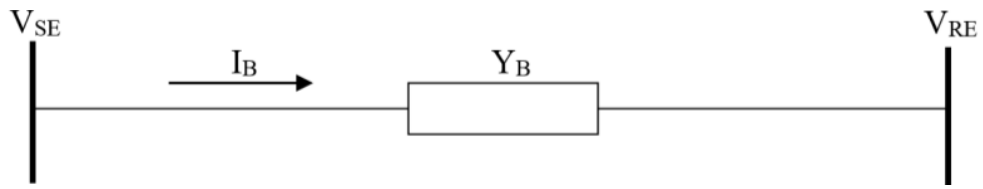

Figure 2. Line section of distribution network

Based on Figure 2, it can be obtained (16).

$$
\boldsymbol{I}_{B}^{a b c}=\boldsymbol{Y}_{B}^{a b c}\left(\boldsymbol{V}_{S E}^{a b c}-\boldsymbol{V}_{R E}^{a b c}\right)
$$

where:

$I_{B}^{a b c}=\left[\begin{array}{lll}I_{B}^{a} & I_{B}^{b} & I_{B}^{c}\end{array}\right]^{T}:$ branch (line) current

$\boldsymbol{V}_{S E}^{a b c}=\left[\begin{array}{lll}V_{S E}^{a} & V_{S E}^{b} & V_{S E}^{c}\end{array}\right]^{T}:$ line sending-end voltage

$V_{S E}^{a b c}=\left[\begin{array}{lll}V_{S E}^{a} & V_{S E}^{b} & V_{S E}^{c}\end{array}\right]^{T}:$ line receiving-end voltage

$Y_{B}^{a b c}:$ three-phase admittance matrix

It is to be noted that the three-phase admittance matrix in (16) is the admittance matrix of the line section in Figure 2. This admittance matrix has the form as (17).

$$
\boldsymbol{Y}_{B}^{a b c}=\left[\begin{array}{lll}
Y_{a a} & Y_{a b} & Y_{a c} \\
Y_{b a} & Y_{b b} & Y_{b c} \\
Y_{c a} & Y_{c b} & Y_{c c}
\end{array}\right]
$$

where $Y_{a a}, Y_{b b}$ and $Y_{c c}$ are self admittances of the line phases; $Y_{a b}, Y_{a c}, Y_{b c}, Y_{b a}, Y_{c a}$ and $Y_{b c}$ are mutual admittances between line phases.

In addition to (16), two other sets of equations are needed to complete the proposed DSLF formulation. These two sets of equations can be derived by examining the condition of nodes of the distribution network as depicted in Figure 3.

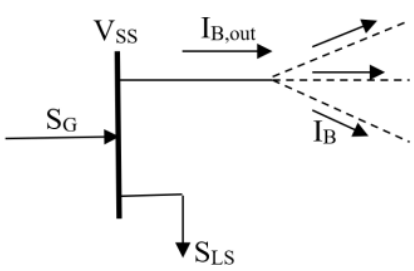

(a)

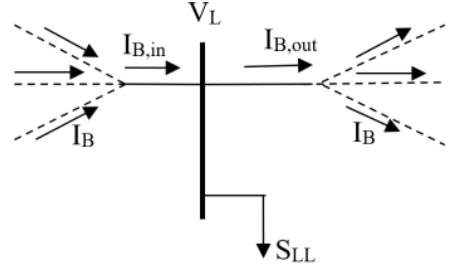

(b)

Figure 3. Conditions at: (a) Substation node, (b) Load node

Based on Figure 3, the two sets of (18) and (19) are obtained.

$$
\begin{aligned}
& S_{G}^{a b c}-S_{L S}^{a b c}=\operatorname{diag}\left(V_{S S}^{a b c}\right)\left(I_{B, \text { out }}^{a b c}\right)^{*} \\
& S_{L L}^{a b c}=\operatorname{diag}\left(V_{L}^{a b c}\right)\left(\boldsymbol{I}_{B, \text { in }}^{a b c}-\boldsymbol{I}_{B, \text { out }}^{a b c}\right)^{*}
\end{aligned}
$$


where:

$S_{G}^{a b c}=\left[\begin{array}{lll}S_{G}^{a} & S_{G}^{b} & S_{G}^{c}\end{array}\right]^{T}$ : power supplied from substation node

$S_{L S}^{a b c}=\left[\begin{array}{lll}S_{L S}^{a} & S_{L S}^{b} & S_{L S}^{c}\end{array}\right]^{T}:$ power demand at substation node

$S_{L L}^{a b c}=\left[\begin{array}{lll}S_{L L}^{a} & S_{L L}^{b} & S_{L L}^{c}\end{array}\right]^{T}:$ power demand at load node

$V_{S S}^{a b c}=\left[\begin{array}{lll}V_{S S}^{a} & V_{S S}^{b} & V_{S S}^{c}\end{array}\right]^{T}:$ substation node voltage

$V_{L}^{a b c}=\left[\begin{array}{lll}V_{L}^{a} & V_{L}^{b} & V_{L}^{c}\end{array}\right]^{T}:$ load node voltage

$I_{B, \text { in }}^{a b c}=\left[\begin{array}{lll}I_{B, \text { in }}^{a} & I_{B, \text { in }}^{b} & I_{B, \text { in }}^{c}\end{array}\right]^{T}:$ branch current entering the node

$I_{B, \text { out }}^{a b c}=\left[I_{B, \text { out }}^{a} \quad I_{B, \text { out }}^{b} \quad I_{B, \text { out }}^{c}\right]^{T}$ : branch current leaving the node

\subsection{Summary of equations and quantities}

This section summarizes all of the sets of nonlinear equations required to solve load flow problem of distribution system installed with three-phase power transformers see in Table 2. All of the electrical quantities (known and unknown) are also summarized in this section see in Table 3.

Table 2. Summary of equations

\begin{tabular}{|c|c|c|}
\hline Set & Set of equations & Number of equations \\
\hline 1 and & $\boldsymbol{I}_{P}^{a b c}-\boldsymbol{Y}_{P P}^{a b c} \boldsymbol{V}_{P}^{a b c}-\boldsymbol{Y}_{P S}^{a b c} \boldsymbol{V}_{S}^{a b c}=\mathbf{0}$ & $\begin{array}{c}6 \times \mathrm{n}_{\mathrm{T}} \\
\left(\mathrm{n}_{\mathrm{T}}: \text { number of transformers }\right)\end{array}$ \\
\hline 1 dind 2 & $\boldsymbol{I}_{S}^{a b c}-\boldsymbol{Y}_{S P}^{a b c} \boldsymbol{V}_{P}^{a b c}-\boldsymbol{Y}_{S S}^{a b c} \boldsymbol{V}_{S}^{a b c}=\mathbf{0}$ & \\
\hline 3 & $\boldsymbol{I}_{B}^{a b c}-\boldsymbol{Y}_{B}^{a b c}\left(\boldsymbol{V}_{S E}^{a b c}-\boldsymbol{V}_{R E}^{a b c}\right)=\mathbf{0}$ & $\begin{array}{c}3 \times \mathrm{n}_{\mathrm{L}} \\
\left(\mathrm{n}_{\mathrm{L}}: \text { number of lines) }\right.\end{array}$ \\
\hline 4 & $S_{G}^{a b c}-S_{L S}^{a b c}-\operatorname{diag}\left(V_{S S}^{a b c}\right)\left(I_{B, \text { out }}^{a b c}\right)^{*}=\mathbf{0}$ & 3 \\
\hline 5 & $\boldsymbol{S}_{L L}^{a b c}-\operatorname{diag}\left(V_{L}^{a b c}\right)\left(\boldsymbol{I}_{B, \text { in }}^{a b c}-\boldsymbol{I}_{B, \text { out }}^{a b c}\right)^{*}=\mathbf{0}$ & $\begin{array}{c}3 \times(n-1) \\
\text { (n: total number of nodes) }\end{array}$ \\
\hline
\end{tabular}

Table 3. Summary of quantities

\begin{tabular}{cll}
\hline Set & Set of known quantities (system data or specified) & \multicolumn{1}{c}{ Set of unknown quantities (to be calculated) } \\
\hline 1 and 2 & $\boldsymbol{Y}_{P P}^{a b c}, \boldsymbol{Y}_{P S}^{a b c}, \boldsymbol{Y}_{S P}^{a b c}, \boldsymbol{Y}_{S S}^{a b c}$ & $\boldsymbol{I}_{P}^{a b c}$ and $\boldsymbol{I}_{P}^{a b c}$ \\
3 & $\boldsymbol{Y}_{B}^{a b c}$ & $\boldsymbol{I}^{a b c}: \boldsymbol{I}_{B}^{a b c}, \boldsymbol{I}_{B, \text { in }}^{a b c}, \boldsymbol{I}_{B, \text { out }}^{a b c}$ \\
4 & $\boldsymbol{V}_{S S}^{a b c}=\left[\begin{array}{lll}1 \angle 0^{o} & 1 \angle-120^{\circ} & 1 \angle 120^{\circ}\end{array}\right]^{T}$ & $\boldsymbol{S}_{G}^{a b c}$ \\
& $\boldsymbol{S}_{L}^{a b c}: \boldsymbol{S}_{L S}^{a b c}, \boldsymbol{S}_{L L}^{a b c}$ & $\boldsymbol{V}^{a b c}: \boldsymbol{V}_{P}^{a b c}, \boldsymbol{V}_{S}^{a b c}, \boldsymbol{V}_{S E}^{a b c}, \boldsymbol{V}_{R E}^{a b c}, \boldsymbol{V}_{L}^{a b c}$ \\
\end{tabular}

\section{VERIFICATION OF THE PROPOSED METHOD}

\subsection{Test system and software conditions}

This section discusses verification and validation of the method proposed in Section 3. IEEE 4-node test system [30] is used in the verification process. It is also to be noted that MATLAB software has been employed to implement the proposed method algorithm. Specifically, MATLAB built-in function $f$ solve has been used to solve the sets of nonlinear equations in Table 2 for the unknown variables described in Table 3.

\subsection{Results and discussion}

Results of the load flow analysis in terms of system voltage profiles are given in Tables 4-12. For the purpose of comparison, results of DSLF analysis from ref. [30] are also presented in the tables. For Y connections, $V_{x}, V_{y}$ and $V_{z}$ are used to indicate $V_{a}, V_{b}$ and $V_{c}$, respectively. On the other hand, for D connections, $V_{x}, V_{y}$ and $V_{z}$ are used to indicate $V_{a b}, V_{b c}$ and $V_{c a}$, respectively. 
Table 4. Voltage profile for GrY - GrY connection

\begin{tabular}{ccccccc}
\hline \multirow{2}{*}{ Node } & \multicolumn{3}{c}{ Proposed method } & \multicolumn{2}{c}{ Ref. [30] } \\
\cline { 2 - 6 } & $V_{x}$ & $V_{y}$ & $V_{z}$ & $V_{x}$ & $V_{y}$ \\
\hline 1 & $7199.6 / 0$ & $7199.6 /-120$ & $7199.6 / 120$ & $7199.6 / 0$ & $7199.6 /-120$ & $7199.6 / 120$ \\
2 & $7106.5 /-0.3$ & $7139.7 /-120.3$ & $7120.8 / 119.6$ & $7107 /-0.3$ & $7140 /-120.3$ & $7121 / 119.6$ \\
3 & $2247.4 /-3.7$ & $2268.5 /-123.5$ & $2255.8 / 116.4$ & $2247.6 /-3.7$ & $2269 /-123.5$ & $2256 / 116.4$ \\
4 & $1917.7 /-9.1$ & $2061.3 /-128.3$ & $1980.7 / 110.9$ & $1918 /-9.1$ & $2061 /-128.3$ & $1981 / 110.9$ \\
\hline
\end{tabular}

Table 5. Voltage profile for D - D connection

\begin{tabular}{ccccccc}
\hline \multirow{2}{*}{ Node } & \multicolumn{3}{c}{ Proposed method } & \multicolumn{3}{c}{ Ref. [30] } \\
\cline { 2 - 6 } & $V_{x}$ & $V_{y}$ & $V_{z}$ & $V_{x}$ & $V_{y}$ \\
\hline 1 & $12470 / 30$ & $12470 /-90$ & $12470 / 150$ & $12470 / 30$ & $12470 /-90$ & $12470 / 150$ \\
2 & $12338.7 / 29.7$ & $12348.6 /-90.4$ & $12321.2 / 149.6$ & $12339 / 29.7$ & $12349 /-90.4$ & $12321 / 149.6$ \\
3 & $3910.6 / 26.5$ & $3914.3 /-93.6$ & $3905 / 146.4$ & $3911 / 26.5$ & $3914 /-93.6$ & $3905 / 146.4$ \\
4 & $3442 / 22.3$ & $3496.9 /-99.4$ & $3383.8 / 140.6$ & $3442 / 22.3$ & $3497 /-99.4$ & $3384 / 140.7$ \\
\hline
\end{tabular}

Table 6. Voltage profile for $\mathrm{Y}-\mathrm{D}$ connection

\begin{tabular}{ccccccc}
\hline \multirow{2}{*}{ Node } & \multicolumn{3}{c}{ Proposed method } & $V_{z}$ & $V_{x}$ & $V_{y}$ \\
\cline { 2 - 6 } & $V_{x}$ & $V_{y}$ & $7199.6 /-120$ & $7199.6 / 120$ & $7199.6 / 0$ & $7199.6 /-120$ \\
\hline 1 & $7199.6 / 0$ & $7130.9 /-120.4$ & $7124.8 / 119.6$ & $7112 /-0.3$ & $7133 /-120.4$ & $7124.6 / 119.6$ \\
2 & $7111.2 /-0.3$ & $3914.8 /-123.6$ & $3909.3 / 116.3$ & $3906 /-3.4$ & $3915 /-123.6$ & $3909 / 116.3$ \\
3 & $3905.7 /-3.5$ & $3496.8 /-129.3$ & $3388.4 / 110.6$ & $3437 /-7.8$ & $3497 /-129.3$ & $3388 / 110.6$ \\
4 & $3437.3 /-7.7$ & &
\end{tabular}

Table 7. Voltage profile for GrY - D connection

\begin{tabular}{ccccccc}
\hline \multirow{2}{*}{ Node } & \multicolumn{3}{c}{ Proposed method } & \multicolumn{3}{c}{ Ref. [30] } \\
\cline { 2 - 6 } & $V_{x}$ & $V_{y}$ & $V_{z}$ & $V_{x}$ & $V_{y}$ \\
\hline 1 & $7199.6 / 0$ & $7199.6 /-120$ & $7199.6 / 120$ & $7199.6 / 0$ & $7199.6 /-120$ & $7199.6 / 120$ \\
2 & $7111.7 /-0.3$ & $7132.1 /-120.3$ & $7123 / 119.6$ & $7113 /-0.3$ & $7132 /-120.3$ & $7123 / 119.6$ \\
3 & $3905.7 /-3.5$ & $3914.9 /-123.6$ & $3909.3 / 116.3$ & $3906 /-3.5$ & $3915 /-123.6$ & $3909 / 116.3$ \\
4 & $3437.3 /-7.8$ & $3496.8 /-129.3$ & $3388.4 / 110.6$ & $3437 /-7.8$ & $3497 /-129.3$ & $3388 / 110.6$ \\
\hline
\end{tabular}

Table 8. Voltage profile for D - GrY connection

\begin{tabular}{|c|c|c|c|c|c|c|}
\hline \multirow{2}{*}{ Node } & \multicolumn{3}{|c|}{ Proposed method } & \multicolumn{3}{|c|}{ Ref. [30] } \\
\hline & $V_{x}$ & $V_{y}$ & $V_{z}$ & $V_{x}$ & $V_{y}$ & $V_{z}$ \\
\hline 1 & $12470 / 30$ & $12470 /-90$ & $12470 / 150$ & $12470 / 30$ & $12470 /-90$ & $12470 / 150$ \\
\hline 2 & $12340.1 / 29.7$ & $12349.1 /-90.4$ & $12318.3 / 149.6$ & $12340 / 29.7$ & $12349 /-90.4$ & $12318 / 149.6$ \\
\hline 3 & $2249.4 /-33.7$ & $2262.9 /-153.4$ & $2259.2 / 86.4$ & $2249 /-33.7$ & $2263 /-153.4$ & $2259 / 86.4$ \\
\hline 4 & $1919.5 /-39.1$ & $2054.1 /-158.3$ & $1986 / 80.9$ & $1920 /-39.1$ & $2054 /-158.3$ & $1986 / 80.9$ \\
\hline
\end{tabular}

Table 9. Voltage profile for $\mathrm{Y}-\mathrm{Y}$ connection

\begin{tabular}{ccccccc}
\hline \multirow{2}{*}{ Node } & \multicolumn{3}{c}{ Proposed method } & \multicolumn{3}{c}{ Ref. [30] } \\
\cline { 2 - 6 } & $V_{x}$ & $V_{y}$ & $V_{z}$ & $V_{x}$ & - \\
\hline 1 & $7199.6 / 0$ & $7199.6 /-120$ & $7199.6 / 120$ & - & - & - \\
2 & $7115.2 /-0.3$ & $7125.9 /-120.4$ & $7126.4 / 119.6$ & - & - & - \\
3 & $2325.2 /-1.2$ & $2136.7 /-123.7$ & $2314.8 / 114.2$ & - & - & - \\
4 & $2017.4 /-5.5$ & $1881.5 /-129.5$ & $2068.1 / 108.7$ & - & - \\
\hline
\end{tabular}

Table 10. Voltage profile for D - Y connection

\begin{tabular}{ccccccc}
\hline \multirow{2}{*}{ Node } & \multicolumn{3}{c}{ Proposed method } & \multicolumn{3}{c}{ Ref. [30] } \\
\cline { 2 - 6 } & $V_{x}$ & $V_{y}$ & $V_{z}$ & $V_{x}$ & $V_{y}$ \\
\hline 1 & $12470 / 30$ & $12470 /-90$ & $12470 / 150$ & - & - & - \\
2 & $12331.7 / 29.7$ & $12350.3 /-90.4$ & $12329.4 / 149.6$ & - & - & - \\
3 & $2339.7 /-31.5$ & $2136.4 /-153.2$ & $2300.9 / 84$ & - & - & - \\
4 & $2033.5 /-35.7$ & $1880.8 /-159$ & $2051.9 / 78.5$ & - & - \\
\hline
\end{tabular}

Table 11. Voltage profile for GrY - Y connection

\begin{tabular}{ccccccc}
\hline \multirow{2}{*}{ Node } & \multicolumn{3}{c}{ Proposed method } & \multicolumn{3}{c}{ Ref. [30] } \\
\cline { 2 - 6 } & $V_{x}$ & $V_{y}$ & $V_{z}$ & $V_{y}$ \\
\hline 1 & $7199.6 / 0$ & $7199.6 /-120$ & $7199.6 / 120$ & - & - & - \\
2 & $7115.7 /-0.3$ & $7127.1 /-120.4$ & $7124.7 / 119.6$ & - & - & - \\
3 & $2325.2 /-1.2$ & $2136.7 /-123.7$ & $2314.9 / 114.2$ & - & - & - \\
4 & $2017.4 /-5.5$ & $1881.5 /-129.5$ & $2068.1 / 108.7$ & - & - \\
\hline
\end{tabular}


Table 12. Voltage profile for $\mathrm{Y}-\mathrm{GrY}$ connection

\begin{tabular}{ccccccc}
\hline \multirow{2}{*}{ Node } & \multicolumn{3}{c}{ Proposed method } & $V_{x}$ & \multicolumn{2}{c}{ Ref. [30] } \\
\cline { 2 - 6 } & $V_{x}$ & $V_{y}$ & $V_{z}$ & - & - \\
\hline 1 & $7199.6 / 0$ & $7199.6 /-120$ & $7199.6 / 120$ & - & - & - \\
2 & $7115.2 /-0.3$ & $7125.9 /-120.4$ & $7126.4 / 119.6$ & - & - & - \\
3 & $2323.2 /-1.1$ & $2132.2 /-123.9$ & $2321.8 / 114.2$ & - & - & - \\
4 & $2017.5 /-5.5$ & $1881.2 /-129.5$ & $2068.4 / 108.7$ & - & - \\
\hline
\end{tabular}

Tables 4-12 show that the results of the proposed method are in close agreement with those given in [30]. However, the method used in [30] requires different set of nonlinear equations for different transformer connections which is not convenient for efficient implementation and incorporation of various transformer connection types. The method proposed in the present paper does not require any special equation to handle the transformer with $\mathrm{D}$ or ungrounded $\mathrm{Y}$ windings.

\section{CONCLUSION}

In this paper, a simple technique or procedure for including three-phase transformer model in distribution system load flow (DSLF) analysis has been proposed. First, a transformer model based on nodal admittance matrix was developed. Then, the model is incorporated into load flow analysis. In the paper, the load flow formulation in terms of branch currents and nodal voltages has also been proposed to enable incorporating the model into the analysis. In the formulation, sets of nonlinear equations are derived at every node and branch of the distribution system by examining the conditions (i.e. electrical quantities relationships). The proposed method can solve the singularity problem, and makes the calculations in forward/backward sweep (FBS) algorithm is easy to be carried out. The results indicate that the proposed method is valid and accurate.

\section{REFERENCES}

[1] B. Sereeter, K. Vuik and C. Witteveen, "Newton power flow methods for unbalanced three-phase distribution networks," Energies, vol. 10, no. 10, pp. 1-20, 2017, doi: 10.3390/en10101658.

[2] A. G. Fonseca, O. L. Tortelli and E. M. Lourenço, "Extended fast decoupled power flow for reconfiguration networks in distribution systems," IET Generation Transmission and Distribution, vol. 12, no. 22, pp. 6033-6040, 2018, doi: 10.1049/iet-gtd.2018.5886.

[3] O. L. Tortelli, E. M. Lourenço, A. V. Garcia and B. C. Pal, "Fast decoupled power flow to emerging distribution systems via complex pu normalization," in IEEE Transactions on Power Systems, vol. 30, no. 3, pp. 1351-1358, May 2015, doi: 10.1109/TPWRS.2014.2343193.

[4] S. Bolognani and S. Zampieri, "On the existence and linear approximation of the power flow solution in power distribution networks," in IEEE Transactions on Power Systems, vol. 31, no. 1, pp. 163-172, Jan. 2016, doi: 10.1109/TPWRS.2015.2395452.

[5] H. Li, H. Zhou, T. Liu and Q. Chen, "A loop-analysis theory based linear power flow method for three-phase distribution power system," in IEEE Access, vol. 7, pp. 157389-157400, Oct. 2019, doi: 10.1109/ACCESS.2019.2949372.

[6] H. Li, X. Yan, J. Yan, A. Zhang and F. Zhang, "A three-phase unbalanced linear power flow solution with PV Bus and ZIP load," in IEEE Access, vol. 7, pp. 138879-138889, 2019, doi: 10.1109/ACCESS.2019.2940229.

[7] H. Ahmadi, J. R. Martı' and A. von Meier, "A linear power flow formulation for three-phase distribution systems," in IEEE Transactions on Power Systems, vol. 31, no. 6, pp. 5012-5021, Nov. 2016, doi: 10.1109/TPWRS.2016.2533540.

[8] A. Garces, "A linear three-phase load flow for power distribution systems," in IEEE Transactions on Power Systems, vol. 31, no. 1, pp. 827-828, Jan. 2016, doi: 10.1109/TPWRS.2015.2394296.

[9] K. Mahmoud and N. Yorino, "Robust quadratic-based BFS power flow method for multi-phase distribution systems," IET Generation Transmission and Distribution, vol. 10, no. 9, pp. 2240-2250, 2016, doi: 10.1049/ietgtd.2015.1518.

[10] S. Petridis, O. Blanas, D. Rakopoulos, F. Stergiopoulos, N. Nikolopoulos and S. Voutetakis, "An efficient backward/forward sweep algorithm for power flow analysis through a novel tree-like structure for unbalanced distribution networks," Energies, vol. 14, pp. 1-20, 2021, doi: 10.3390/en14040897.

[11] Y. Ju, W. Wu, B. Zhang and H. Sun, "An extension of FBS three-phase power flow for handling PV nodes in active distribution networks," in IEEE Transactions on Smart Grid, vol. 5, no. 4, pp. 1547-1555, Jul. 2014, doi: 10.1109/TSG.2014.2310459.

[12] G. W. Chang, S. Y. Chu and H. L. Wang, "An Improved backward/forward sweep load flow algorithm for radial distribution systems," in IEEE Transactions on Power Systems, vol. 22, no. 2, pp. 882-884, May 2007, doi: 10.1109/TPWRS.2007.894848.

[13] U. Ghatak and V. Mukherjee, An improved load flow technique based on load current injection for modern distribution system," Electrical Power and Energy Systems, vol. 84, pp. 168-181, Jan. 2017, doi: 10.1016/j.ijepes.2016.05.008.

A new method to incorporate three-phase power transformer model into distribution ... (Rudy Gianto) 
[14] T.-H Chen, W.-C Yang, T.-Y Guo and G.-C Pu, "Modeling and analysis of asymmetrical three-phase distribution transformer banks with mid-tap connected to the secondary neutral conductor," Electric Power Systems Research, vol. 54, no. 2, pp. 83-89, May 2000, doi: 10.1016/S0378-7796(99)00075-9.

[15] S. S. Moorthy and D. Hoadley, "A new phase-coordinate transformer model for Ybus analysis," in IEEE Transactions on Power Systems, vol. 17, no. 4, pp. 951-956, Nov. 2002, doi: 10.1109/TPWRS.2002.804996.

[16] M. R. Irving and A. K. Al-Othman, "Admittance matrix models of three-phase transformers with various neutral grounding configurations," in IEEE Transactions on Power Systems, vol. 18, no. 3, pp. 1210-1212, Aug. 2003, doi: 10.1109/TPWRS.2003.814905.

[17] Z. Wang, F. Chen and J. Li, "Implementing transformer nodal admittance matrices into backward/forward sweepbased power flow analysis for unbalanced radial distribution systems," in IEEE Transactions on Power Systems, vol. 19, no. 4, pp. 1831-1836, Nov. 2004, doi: 10.1109/TPWRS.2004.835659.

[18] P. Xiao, D. C. Yu and W. Yan, "A unified three-phase transformer model for distribution load flow calculations," in IEEE Transactions on Power Systems, vol. 21, no. 1, pp. 153-159, Feb. 2006, doi: 10.1109/TPWRS.2005.857847.

[19] R. Verma and V. Sarkar, "Active distribution network load flow analysis through non-repetitive FBS iterations with integrated DG and transformer modeling," IET Generation Transmission and Distribution, vol. 13, no. 4, pp. 478484, Jan. 2019, doi: 10.1049/iet-gtd.2018.5478.

[20] U. Ghatak and V. Mukherjee, "A fast and efficient load flow technique for unbalanced distribution system," Electrical Power and Energy Systems, vol. 84, pp. 99-110, 2017, doi: 10.1016/j.ijepes.2016.05.002.

[21] M. Bazrafshan and N. Gatsis, "Comprehensive modeling of three-phase distribution systems via the bus admittance matrix," in IEEE Transactions on Power Systems, vol. 33, no. 2, pp. 2015-2029, Mar. 2018, doi: 10.1109/TPWRS.2017.2728618.

[22] M. Dilek, F. de Leon, R. Broadwater and S. Lee, "A robust multiphase power flow for general distribution networks," in IEEE Transactions on Power Systems, vol. 25, no. 2, pp. 760-768, May 2010, doi: 10.1109/TPWRS.2009.2036791.

[23] A. Carreno, M. Perez, C. Baier, A. Huang, S. Rajendran and M. Malinowski, "Configurations, power topologies and applications of hybrid distribution transformers," Energies, vol. 14, pp. 1-35, 2021, doi: 10.3390/en14051215.

[24] S. Claeys, G. Deconinck and F. Geth, "Decomposition of n-winding transformer for unbalanced optimal power flow," IET Generation Transmission and Distribution, vol. 14, no. 24, pp. 5961-5969, 2020.

[25] U. Eminoglu and M. H. Hocaoglu, "Three-phase transformer modeling for forward/backward sweep-based distribution systems power flow algorithms," 2007 42nd International Universities Power Engineering Conference, 2007, pp. 115-120, doi: 10.1109/UPEC.2007.4468930.

[26] U. Eminoglu and M. H. Hocaoglu, "Three-phase transformer and voltage regulator modeling for backward/forward sweep-based distribution system power flow algorithms," International Journal of Electrical Engineering Education, vol. 46, no. 1, pp. 30-46, 2009, doi: 10.7227/IJEEE.46.1.3.

[27] M. Abdel-Akher, and K. Mahmoud, "Implementation of three-phase transformer model in radial load-flow analysis," Ain Shams Engineering Journal, vol. 4, pp. 65-73, Mar. 2013, doi: 10.1016/j.asej.2012.04.009.

[28] I. Džafić, R. A. Jabr and H. Neisius, "Transformer modeling for three-phase distribution network analysis," in IEEE Transactions on Power Systems, vol. 30, no. 5, pp. 2604-2611, Sept. 2015, doi: 10.1109/TPWRS.2014.2360812.

[29] W. H. Kersting, Distribution system modeling and analysis, $4^{\text {th }}$ ed., Boca Raton: CRC Press, 2017, doi: $10.1201 / 9781315120782$.

[30] IEEE PES Distribution System Analysis Subcommittee, IEEE 4 Node Test Feeder: The Institute of Electrical and Electronic Engineers Inc., 2006.

\section{BIOGRAPHIES OF AUTHORS}

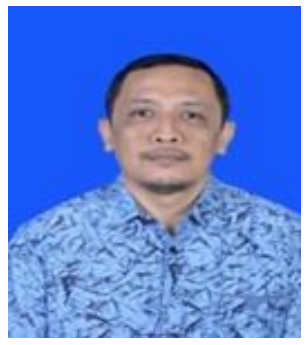

Rudy Gianto received BE, ME and PhD degrees from Universitas Tanjungpura in 1991, Institut Teknologi Bandung in 1995, and The University of Western Australia in 2009 respectively. Currently, he is an Associate Professor at Universitas Tanjungpura, Indonesia. He has published several international journal and conference papers. His research interests include power system analysis, simulation of power system dynamics, and distributed generation.

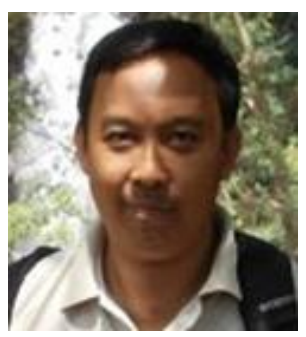

Purwoharjono received BE, ME and Doctor degrees from Universitas Tanjungpura in 1997, Institut Teknologi Sepuluh November in 2001, and 2014 respectively. Currently, he is an Associate Professor at Universitas Tanjungpura, Indonesia. He has published several international journal and conference papers. His research interests include power flow analysis, and system optimization. 